\title{
Legislation pertaining to rare diseases: genetic counseling, ethics, practitioner-patient relationship
}

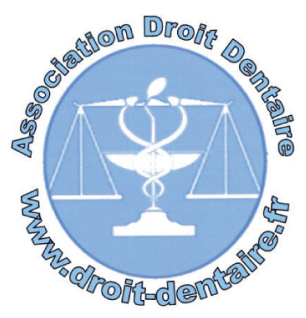

\author{
Patrick LEVIGNE, Christophe CORDIER, \\ Agnès BLOCH-ZUPAN
}

\begin{abstract}
The development of predictive medicine for dentistry is very promising but there are potential abuses as well. Given that genetic testing is at the forefront of predictive medicine, legislation has been established governing activity related to genetics that is both open to the advances that genetic testing can provide and, at the same time, respectful of the fundamental rights of patients and in particular of the rights of minors.
\end{abstract}

\section{KEY WORDS}

Bioethics

Consent
Genetics

Genetic testing

\section{INTRODUCTION}

The 7000 rare diseases known to date by definition affect fewer than 1 in 2000 people: more than 900 of these diseases include orofacial manifestations in their clinical picture (dental abnormalities in number, shape, size, structure of mineralized tissue, eruption, resorption...not counting associated dysmorphias of the basal bone), and these manifestations are either isolated or combined with other symptoms in the context of syndromes ${ }^{3}$.

These patients, treated at referral centers for orofacial rare diseases and at the member diagnostic and treatment centers, benefit from a thorough "customized guidance" to medical genetic services with

Address for correspondence:

\section{P. LEVIGNE}


the objective of including orofacial abnormalities within the family medical history of the patient, to propose and to help determine the diagnosis, and to benefit from genetic testing when available, from genetic counseling and from a coordinated, comprehensive treatment plan.

In fact, it is always a delicate issue when the dental surgeon deals with questions related to genetic diseases during consultation. The dental surgeon has the duty to identify orofacial abnormalities, to recognize the genetic nature of these anomalies and to direct patients to appropriate treatment facilities such as centers specialized in orofacial treatment or referral centers for treatment and diagnosis and/or genetic counseling. These core competencies are cited in an article intended to articulate the roles and areas of expertise of healthcare professionals in Europe as well as the various means of training in genomic medicine ${ }^{13}$.

The partnerships between dental surgeons practicing in private practice and the objectives of the hospital and university medical centers dedicated to rare diseases are not only to improve treatment management of patients and their families based on the latest scientific data but also to actively participate in the national endeavor to know and to recognize

\section{1 - LEGISLATIVE FRAMEWORK}

Rare diseases have been recognized as one of the five top priorities that requires a strategic plan, as stated in the public health policy adopted on August 9, 2004. A na- these diseases and their specific orodental manifestations using census data in specialized registries (D4/phenodent, ww.phenodent.org), as well as the opportunity for patients and their families to participate in research programs.

Advances in genetics lead to the identification of new rare diseases, of new genes, and to the recognition of associated symptoms (amelogenesis imperfecta and nephrocalcinosis for example) a disease for which it is now possible to make a genetic diagnosis and to provide adaptive followup treatment. Good practice recommendations and clinical treatment management guidelines have been published $^{11}$.

Research programs, such as the INTERREG IV/offensive Sciences A27, aim to correlate genotypes with phenotypes for orofacial manifestations of rare diseases ${ }^{7}$ with the objective of creating diagnostic genetic tools targeted towards orodental anomalies that subsequently helps to improve treatment plans ((http:// download. steinbeis-europa.de/tmo-wo/ OFFENSIVESSCIENCESABZ2012DEF.pdf).

In this extremely dynamic and sensitive context ${ }^{1,2,5,6,8,9,12,14,15}$, we thought it was important to address the legal aspects of rare diseases particularly in regard to genetic testing.

tional plan was launched at the end of 2004 for the years 2005-2008. One of the objectives of this first plan for rare diseases was to improve the organization of screening 
programs as well as access to diagnostic testing and to genetic counseling, given that $80 \%$ of rare diseases are genetic in origin (the rest are divided between rare infectious diseases and auto-immune diseases). Additionally, a general organization of screening tests as defined from a legal perspective was established following the decree $n^{\circ}$ 2008-321 on April 4, 2008 relating to the examination of human genetic characteristics and to the identification of the person using DNA for medical purposes.

These genetic tests are offered by healthcare professionals, medical doctors board certified in genetics and/or genetic counseling (with a Master's degree in human pathology, specialty in "genetic counseling and predictive medicine"). These individuals with a Master's degree provide services that are prescribed by a medical doctor and under the responsibility of one or more medical geneticists. The genetic analyses are performed by pathologists certified in genetics who have been accredited by the Agency of Biomedicine. These doctors are the only accredited providers of testing for medical purposes (Art. L 1131-3 of the Public Health Code pursuant to legislation introduced on August 6, 2004). The new profession of genetic counselors is forthwith a part of the transfer of tasks and of core competencies process, fostering collaboration among healthcare professionals, as members of the treatment team, as Professor Yvon Berland had suggested during his reports on medical demography in France (2002-2003). The healthcare profession of genetic counselor was created by decree $n^{\circ} 2007-1429$ on $10 / 3 / 2007$ within the framework of legislation for public health 2004 (Art. L 1132-1 of the law 2004-806 on 8/6/2004); the medical specialty of genetic medicine had already been established by article 2 of the law on February 4, 1995.

\section{Work elements relating to genetics are codified:}

Legal documents concerning the examination of human genetic characteristics describe with some restrictions the ultimate purpose of these tests that can only be performed for some medical objective or for scientific research, any other use is strictly prohibited in the context of the workplace or for medical insurance purposes.

The Civil Code allots 4 articles to "the examination of human genetic characteristics"; these provisions initiated by law n94-653 on July 29, 1994 relating to the protection of the human body were modified by law $n^{\circ}$ 2004-800 on August 6, 2004 relative to bioethics:

- Art. 16-10: The genetic study of human characteristics cannot be undertaken except for medical reasons or for scientific research. The consent of the individual must be obtained before the testing is performed.

- Art. 16-11: The identification of an individual using DNA is only permitted when the process is part of an investigation or judicial enquiry or instructions implementing a judicial procedure, or for scientific research. When the identification is performed for medical purposes or for scientific research, the informed consent of 
the individual must be obtained beforehand.

- Art. 16-12: Only certified healthcare personnel designated by the accreditation Counsel who meet the conditions laid out by the decree of the State Counsel can process identifications with DNA.

- Art. 16-13: No one can be discriminated against based on genetic characteristics.

The Public Health Code contains several articles related to predictive medicine, to genetic research, to genetic identification and to the profession of genetic counselor (Art. L. 1131-1 to L. 1132-2).

- Art. L. 1131-1: The examination of human genetic characteristics may only take place for medical purposes or for scientific research and only after informed consent is obtained. When the examination is performed for medical purposes, informed consent must be obtained in writing. Examinations for scientific purposes are regulated by the provisions of title II of this book. Only in exceptional cases, when this study is undertaken for medical purposes, the informed consent of the individual may be dispensed with in the interest of the individual and in order to respect confidentiality. Subject to the same conditions, the informed consent of the individual may also be dispensed with when the identification of an individual using DNA is sought for medical reasons.

The new Penal Code presents a series of infractions related to activity in genetics, for example the act of diverting from their medical purposes or from scientific research information collected about individuals by a study of their genetic characteristics (Art. 226-25 to 226-30) and the Public Health Code takes up these same issues (Art. L. 1133-1 to 1133-5).

The August 6, 2004 legislation relative to bioethics replaced the expression "genetic study of the characteristics of an individual" with "examination of the genetic characteristics of an individual", the law revised the modalities of informed consent for this examination.

\section{2 - PATIENT-PRACTITIONER RELATIONSHIP, INFORMATION, INFORMED CONSENT}

The second plan for rare diseases (2011-2014) reconfirms the organization of screening tests and insists on the need for professionals and the larger public to be better informed about screening and related issues, in order to have top to bottom information flow between scholarly institutions, hospitals, local practitioners and patients in order to avoid a false diagnosis that is always detrimental.
Before DNA testing, It is necessary to obtain the free and informed consent form from the individual. (Art. L. 1131-1. C. Public Health: Art. 16-10 C. Civil). The manner in which the consent is obtained is always in writing and cosigned by the patient who is receiving the information and by the certified medical geneticist and/or the genetic counselor who have fully explained all 
aspects of genetic testing as well as the purpose of the testing (the patient must be informed about the nature and the purpose of the testing and about possible implications of the results). The written consent of the individual specifically states the purpose of the testing that can be revoked at any time. Genetic identification is one the rare medical situations along with others such as organ donation, medically assisted reproduction or biomedical research where it is necessary to obtain written consent, whereas in other cases of health care procedures there is no need for this formality because the burden of proof may be established by other means, therefore written acknowledgement is no longer mandatory. The patient can express in writing, his wish to remain uninformed of the diagnosis (Art. L. 11112 al 4. C. public health): "the wish of an individual to remain uninformed of a diagnosis must be respected, except when others are exposed to the risk of transmission".

Testing genetic characteristics for medical purposes is intended:

- either to confirm or exclude a diagnosis of a genetic disease in a person who presents its symptoms;

- or to determine whether an asymptomatic individual, has the characteristics of one or more genes that are susceptible to the future development of a disease in the individual or his offspring.

However, if it is impossible to obtain the consent of the individual or to consult with the confidential advisor, the legal guardian or a member of the family, the testing may be performed for medical purposes in the individual's interests (Art. L. 1131-1, al. 2. C. public health).

The results of genetic testing should not be directly communicated to the patient by the laboratory that performs the analysis but by the prescriber: either a certified medical geneticist, or a genetic counselor who is aware of the clinical situation (the disease, therapeutic treatment plan) and the consequences for the family and is capable of interpreting the results of the test. This doctor must work in conjunction with a clinical genetics team ("Rules for good practice in constitutional genetics for medical purposes" ABM and HAS 2013).

How test results are provided must be defined beforehand and in particular during the consultation that led to the prescription for testing (medical doctor or a genetic counselor under the responsibility of medical geneticist).

In every case, psychological counseling should be made readily available.

A recommendation from the French National Authority for Health (HAS) dated May, 2012 deals with the "provision of health-related information to an individual" and is intended to be a practical application of the law concerning disclosure of information: therefore, it is recommended that the information provided be understandable for the individual, and customized to the patient, not just general information, delivered verbally, the written information is only in addition to the discussion with the patient. The information alone has become a medical procedure that then helps the patient make a decision. 
However, specific provisions are reserved that allow for DNA testing in the legitimate interest of the family, when the benefit for the individual involved is the basic condition. The uniqueness of a genetic diagnosis stems from it being a definitive diagnosis, that concerns not just the patient but all his blood relatives. In fact, the question then arises as to whether it is necessary to inform the family of the individual who has undergone testing for genetic characteristics, when there appears some risk that the family might be infected by keeping medical secrets. Additionally, when the bioethics law was revised in 2004, a legislator tried to create a policy that made it possible to alert blood relatives of the concerned patient (in some ways, like information about the family) that would be provided to the French Biomedicine Agency so as to avoid forcing the medical subscriber to divulge the professional secret, but this law never came into being, since no directives were given as to how it should be applied. However, when bioethics laws were revised in 2011, legislators decided to encourage healthcare workers when in consultation with patients to convince them to tell their families themselves in case of a genetic diagnosis subsequent to DNA testing that poses a serious risk, the doctor informs the patient of the consequences that his silence could impact other members of the family who are potentially affected by the disease which may be treated. Given that every consultation always begins with a family history, if the patient refuses to reveal the diagnosis to the members of the family, the law is clear: it makes a provision for the healthcare professional to send a letter to the members of the family, indicating that someone (without naming the individual) in the family circle has a genetic disease that should be treated or subject to genetic counseling, and a list of counselors is included with the letter. Therefore, the patient is required to inform his relatives, if he doesn't do it himself he cannot object if the treating doctor does.

More recently and in relation to informing the relatives, the legislature provided a framework for this procedure with the Law $\mathrm{n}^{\circ}$ 2013-527 on June 20,2013 relative to the conditions of conveying the information to the relatives when it involves testing for human genetic characteristics for medical purposes... This mandate seems necessary given the progress made by advances in genetics with the advent of the new generation of sequencing technologies.

Patients have to change their way of thinking, they should be told that a genetic disease is not an embarrassing disease, healthcare professionals must change also because they must see that in addition to the patient, the impact of a genetic disease extends to the whole family.

- Testing for the benefit of the members of a family: the testing can be performed on a person for the benefit of the family and not just for the patient provided that the provisions of the protocol are followed, especially those dealing with free and informed consent, and that the members of the family involved draw some benefit from it for prevention, diagnosis or treatment therapy. 
- If testing is planned for a minor without legal capacity to consent, the testing must be deferred until the minor has reached legal capacity, unless the delay will harm the minor's health or psychological equilibrium, then parental authority should benefit from the same information as the individual capable of giving consent; since the opinion of the minor is increasingly being taken into consideration in proportion to his or her age and degree of maturity, the minor should receive information appropriate for his/her level of understanding because he, himself, has the right to receive this information and therefore to participate in the decision making process even if the consent is still required from the guardians.

Art. L. 1111-4 al. 5. C. Public Health: "The consent of the minor should systematically be requested if he or she is able to express his or her will and participate in the decision making process". The minor's will-

\section{3- ETHICS AND RESEARCH}

While it is true that bioethics covers questions of medical ethics, beyond the code of conduct it implies a reassessment of the evolution of a society driven by scientific developments. Scientific and technological advances circle the world at the speed of the internet, medical research is becoming internationalized and the need to establish some universal ethical guidelines increasingly is the topic of planetary debate.

Internationally, in the spirit of the Universal Declaration of Human ingness to cooperate is required given that the consent of the parents alone has no legal value. A qualified person must be available to answer any possible questions from the legal representative or from the individual undergoing the test.

Quite recently, the legislature noted (Decree on May 27, 2013 defining the rules of good practice applicable to the examination of human genetic characteristics of an individual for medical purposes): "The individual must be the focus of concern of all who play a role in the diagnosis of genetic diseases. This is why the information, the consent and the manner in which the results are delivered must be central elements in how the genetic study is conducted. The genetic testing should only be prescribed when they are clinically useful and the individual wants to be tested. The fact that a test is available and can be provided does not justify its prescription or implementation".

Rights proclaimed by general assembly of the United Nations on December 10, 1948 in reaction to the atrocities committed by Nazi Germany (directly inspired by the Declaration of the rights of man and of the citizen in 1798); UNESCO by way of the International Committee on Bioethics (ICB), that is the only international statement concerning the domain of bioethics, contributed to the elaboration of a fundamental document on bioethics with the Universal Declaration concerning the human genome and human rights 
in 1997 that was taken up again by the General Assembly of the United Nations in 1998, therefore becoming the first universal position paper on bioethics that establishes a framework for research in the domain of genetics. Although certain nations have already made laws and regulations pertaining to bioethics intended to protect human dignity, many other countries that presently do not have a legislative and regulatory framework in this area wanted to use the guidelines laid out in the Universal Declaration of human rights adopted by UNESCO on October 19, 2005.

In Europe, the European Council, an institution created in 1949 for many reasons and specifically for the purpose of promoting respect for its Convention for protecting human rights and basic liberties from November 4, 1950 (with a political objective, for the establishment of democracy following the totalitarian regimes of World War II), that makes direct reference to the United Nations' Universal Declaration of human rights, stated that in the field of medicine there was a development in human genetics the attracted the attention of the European Council concerning the protection of these rights and liberties given the foreseeable development and possible risk of eugenics. Consequently, a group of experts in bioethics was assembled to examine the problems that are part of more extensive application. The group of experts became the Governing Committee for Bioethics (GCB) that wrote the Convention on human rights and biomedicine on April 4, 1997 at Oviedo (Spain).
The Convention of Oviedo contains the basic principles for the uses of medical and biological procedures for humans.

This convention and additional protocols are part of international law that regulates the application of medicine and biology, including human subject research, with binding internal legal power after ratification by the member states; France had to wait 14 years and on April 1, 2012 the convention of Oviedo entered into force there. The Convention of Oviedo and additional protocol adopted on May 7, 2008 "Biomedical research relating to genetic testing for medical purposes" provides the specific enforceable framework for any biomedical research. These two documents contain typical rules such as the need for free and informed consent from the concerned individual, the primacy of human beings, the respect of norms and professional ethics and rules of conduct that therefore protect the afflicted individuals involved in a research project by doing a risk benefit analysis. A general principle is that this research cannot be undertaken unless there is no alternative and unless there is no disproportion between the risks incurred and the expected benefits; in cases where the research has no potential benefit for the patient, the research project therefore must not present any element of risk or obligation. For minors, this protection is reinforced, the expected results of a scientific research project must include a direct and real benefit for their health. Authorization must be given by the legal guardian or an 
authority designated by national law. No research can be undertaken without the agreement of a competent national authority as it relates to the scientific pertinence of the project and the ethical issues involved.

On a national level, legislation dealing with bioethics affirms the general principles of the primacy of the individual and protects him in various healthcare-related situations.

The first laws of bioethics:

- the July 1, 1994 law has to do with how personal data is treated when the purpose of the research falls into the category of health;

- the two other laws passed on July 29, 1994, the first with regard to the human body and the second deals with donations and use of elements and products of the human body as well as prenatal diagnosis and medical assistance for procreation.

The process for revising bioethics law led to the law dated August 6, 2004, the major themes of the reform dealt with cloning for therapeutic purposes, with experimenting with new techniques for assisted reproduction; and also the creation of the Agency for Biomedicine whose missions have been outlined in Article L. 1418-1. C. Public Health, for example, a mission to assist health professionals by disseminating recommendations for best practices. Additionally, in the area of genetics, the recommendations from the Agency for Biomedicine discuss the reception of the patients, the way to talk to them about the genetic disease and to share the results with them especially when they are difficult to interpret. The Agency for Biomedicine also has the mission to assess the work elements that it provides, this is particularly important in the field of genetics that presents serious health and ethical risks. One of agency's missions is to inform parliament and the government about its areas of expertise (such as human genetics).

The law of bioethics dated July 7,2011 , is the result of the revision clause written in the 2004 law and should be investigated by members of parliament dealing with the following points: child law - prenatal diagnosis and genetic data - use of the human body for medical purposes and research - the emergence of neuroscience and nanotechnology.

France is the first country in Europe to have such comprehensive legislation.

\section{CONCLUSION}

The National Consultative Committee on Ethics (CCNE) in its notice $n^{\circ}$ 120 on April 25, 2013 stresses the need for information and for improved management of treatment for the association for persons with disabilities or with genetic diseases, and is deeply concerned by the extremely inadequate research dedicated to disabilities. The dental surgeon, can, while fully cognizant of the legal and ethical issues, participate and encourage his patients to take part in targeted research programs. 


\section{REFERENCES}

1. Ayme S, Gribaldo L, Matthijs G, Borry P. European Workshop on Genetic Testing Offer in Europe (19-20 November 2012, Ispra, Italy). JRC Scientific and Policy Reports, EuroGentest European Commission, 2013.

2. Ayme S, Matthijs G, Soini S. Patenting and licensing in genetic testing : recommendations of the European Society of Human Genetics. Eur J Hum Genet 2008;16, Suppl 1:S10-19.

3. Bloch-Zupan A, Sedano H, Scully C. Dento/Oro/Craniofacial Anomalies and Genetics. London: Elsevier Inc. 2012.

4. Bloch-Zupan A, Jamet X, Etard C, Laugel V, Muller J, et al. Homozygosity mapping and candidate prioritization identify mutations, missed by whole-exome sequencing, in SMOC2, causing major dental developmental defects. Am J Hum Genet 2011;89:773-781.

5. Borry P, Evers-Kiebooms G, Cornel MC, Clarke A, Dierickx K. Genetic testing in asymptomatic minors: background considerations towards ESHG Recommendations. Eur J Hum Genet 2009;17:711-719.

6. Consortium Irdr. Policies and guidelines, 2013. IRDiRC www.irdirc.org.

7. Gasse B, Karayigit E, Mathieu E, Jung S, Garret A, et al. Homozygous and Compound Heterozygous MMP20 Mutations in Amelogenesis Imperfecta. J Dent Res 2013.

8. Godard B, Kaariainen H, Kristoffersson U, Tranebjaerg L, Coviello D, et al. Provision of genetic services in Europe: current practices and issues. Eur J Hum Genet 2003;11 Suppl 2:S13-48.

9. Hastings $R$, de Wert G, Fowler B, Krawczak M, Vermeulen $E$, et al. The changing landscape of genetic testing and its impact on clinical and laboratory services and research in Europe. Eur J Hum Genet 2012;20:911-916.

10. Jaureguiberry G, De la Dure-Molla M, Parry D, Quentric M, Himmerkus N, et al. Nephrocalcinosis (Enamel Renal Syndrome) Caused by Autosomal Recessive FAM20A Mutations. Nephron Physiol 2013;122:1-6.

11. Mornet E, Beck C, Bloch-Zupan A, Girschick H, Le Merrer M. Clinical utility gene card : hypophosphatasia. Eur J Hum Genet 2011;19.

12. Patenting and licensing in genetic testing. Eur J Hum Genet 2008;16:405-411.

13. Skirton H, Lewis C, Kent A, Coviello DA. Genetic education and the challenge of genomic medicine : development of core competences to support preparation of health professionals in Europe. Eur J Hum Genet 2010;18:972-977.

14. Statement of the ESHG on direct-to-consumer genetic testing for health-related purposes. Eur J Hum Genet 2010;18:1271-1273.

15. Van El CG, Cornel MC, Borry P, Hastings RJ, Fellmann F, et al. Whole-genome sequencing in health care: recommendations of the European Society of Human Genetics. Eur J Hum Genet 2013;21:580-584. 